\title{
Inferior Vena Cava Filter Insertion
}

\author{
Brian Funaki, M.D. ${ }^{1}$
}

Inferior vena cava filtration is a common albeit controversial practice. Filter insertion varies widely by hospital. In my practice, nearly all patients with deep venous thrombosis or pulmonary embolism who are unable to undergo anticoagulation receive an inferior vena cava filter. Retrievable filters are also placed prophylactically in high-risk patients prior to elective surgery.

\section{PROCEDURE}

\section{Preprocedure}

The patient should have deep venous thrombosis or pulmonary embolism documented by an imaging study with failure of or contraindication to anticoagulation. A permanent or retrievable filter should be chosen. Currently in my practice, retrievable filters are used liberally because these can simply be left in place as a permanent filter if later retrieval is not indicated.

\section{Puncture}

After subcutaneous lidocaine is administered, either the right or left common femoral vein or right or left internal jugular vein is used for venopuncture (the right veins tend to be better choices as they provide a straight path into the inferior vena cava). I often attach the micropuncture (21-gauge) needle to the syringe containing lidocaine to anesthetize the deeper tissues and perform venopuncture concurrently with administration of local anesthesia. If the jugular vein is used for access, ultrasound guidance is used for venopuncture. If the femoral veins are used for access, it is generally advisable to puncture on the side opposite the deep venous thrombosis.

\section{Wire Purchase}

Next, a 0.018 -inch guide wire is advanced into the vein and the needle is exchanged over this guide wire for coaxial 3- and 5-French dilators included in the kit. The 0.018 -inch guide wire and 3 -French dilator are removed. A 0.035 -inch guide wire is then advanced through the 5French dilator and the dilator is exchanged for a pigtail catheter, which is positioned at the junction of common iliac veins.

\section{Venacavography}

A venacavogram is then performed by injecting $30 \mathrm{~mL}$ of contrast at $20 \mathrm{~mL}$ per second (Fig. 1). The study is then evaluated with the following questions in mind:

- Is there clot in the cava? The presence of clot may require the filter to be placed above the renal veins or may preclude placement altogether.

- What is the diameter of the cava? Larger-diameter vena cavae (between 30 and $40 \mathrm{~mm}$ ) may require a Bird's Nest filter (Cook, Bloomington, IN, USA) or bilateral iliac vein filters $(>40 \mathrm{~mm}$ ).

- Where are the renal veins? Ideally, the filter should be placed below the lowest renal vein. In the case of a circumaortic left renal vein, placing the filter below or across the lower moiety is necessary.

- Are there signs of anatomic variation? Inflow should be seen from the contralateral iliac vein and both renal veins. If inflow is not identified in the contralateral
${ }^{1}$ Section of Vascular and Interventional Radiology, University of Chicago Hospitals, Chicago, Illinois.

Address for correspondence and reprint requests: Brian Funaki, M.D., Section of Vascular and Interventional Radiology, University of Chicago Hospitals, 5840 S. Maryland Avenue, MC 2026, Chicago, IL 60637.
Clinical Management Issues in Interventional Radiology; Guest Editor, H. Bob Smouse, M.D.

Semin Intervent Radiol 2006;23:357-360. Copyright (C) 2006 by Thieme Medical Publishers, Inc., 333 Seventh Avenue, New York, NY 10001, USA. Tel: +1(212) 584-4662.

DOI 10.1055/s-2006-957026. ISSN 0739-9529. 
iliac vein, this indicates either a deep venous thrombosis or a duplicated inferior vena cava. In the case of the latter, filters must be placed in both cavae.

\section{Filter Insertion}

The pigtail catheter is then exchanged for a filter deployment sheath over a stiff 0.035 -inch guide wire such as an Amplatz wire. If one of the femoral veins has been
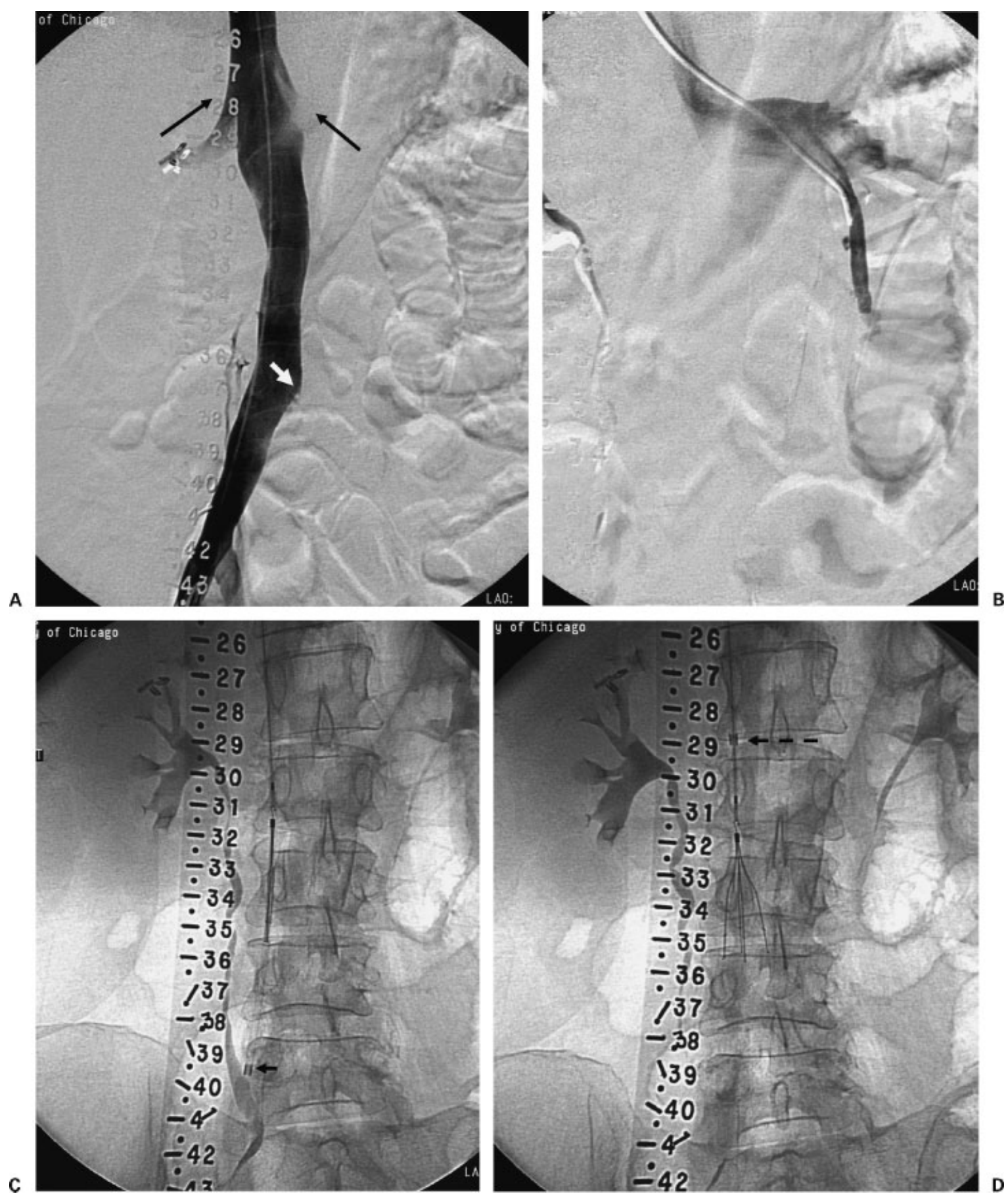

Figure 1 Insertion of Tulip inferior vena cava filter via right internal jugular vein approach. (A) Inferior vena cavagram shows normalcaliber inferior vena cava with inflow from both renal veins (black arrows) and questionable inflow from the left common iliac vein (white arrow). This indicates either left-sided deep venous thrombosis or cava duplication. (B) Selective left renal venogram shows opacification of the left gonadal vein and renal vein without evidence of cava duplication. (C) Fluoroscopic image shows filter advanced in deployment sheath to the level of the infrarenal inferior vena cava. Note tip of sheath is far below level of renal veins (arrow). (D) Fluoroscopic image shows filter unsheathed but still attached by apical hook. 


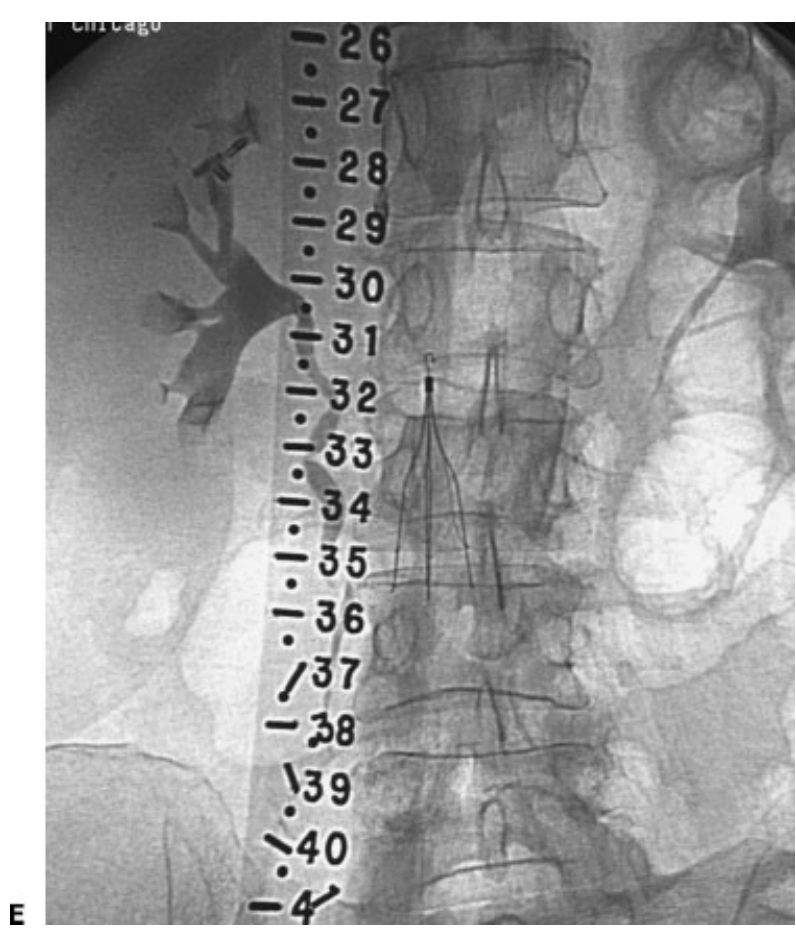

Figure 1 (con't) (E) Final fluoroscopic image after deployment shows infrarenal filter in good position without evidence of tilting.

catheterized, the pigtail catheter should be advanced into the upper inferior vena cava prior to pushing the wire through the pigtail. Similarly, if a jugular vein has been catheterized, the pigtail catheter is pushed into the lower inferior vena cava prior to guide wire insertion. This maneuver prevents the wire from accidentally engaging a paraspinal vein or one of the renal veins (see Morbidity and Mortality Case in Semin Intervent Radiol 2006;23:298-302).

There are a variety of filters available but most permanent (LP Venatech, B Braun, Evanston, IL, USA; Simon Nitinol, Bard, Covington, GA, USA; Greenfield-Boston Scientific, Natick, MA, USA; TrapEase, Cordis, Miami, FL, USA) and retrievable (Tulip, Cook; G2, Bard) filters have similar deployment mechanisms. In nearly all cases, the filter should be positioned within the deployment sheath in the desired location (which is typically immediately below the inflow of renal veins). Then, instead of pushing the filter out of the sheath, the sheath is retracted while the filter is held in craniocaudal position. When the filter is completely out of the sheath, it will spontaneously expand in position. Some devices such as the jugular Tulip filter need to be released with a push-button trigger; in other cases (e.g., Venatech), simply releasing the filter from the sheath results in deployment. Each filter has characteristic idiosyncrasies. For example, when releasing the Tulip via a jugular approach, it is helpful to apply gentle back-tension on the filter (by retracting the device) and simultaneously pushing the trigger. If this is done improperly, the release hook will push the filter forward, resulting in tilting. Tilted filters are more difficult (and sometimes impossible) to retrieve, especially if the apical hook contacts the cava wall.

\section{DISCUSSION}

To further illustrate the procedure, it is helpful to analyze what can go wrong. Acutely, most complications relate to difficulty in access or malposition of the filter. Malposition can occur for a variety of reasons including operator error (e.g., the filter is deployed by pushing it out of the sheath instead of retracting the sheath and allowing filter to expand) or anatomic variation (e.g., placement in a paraspinal vein). It is important to remember that malposition in the renal veins usually occurs from a jugular approach when the filter sheath is inadvertently pushed into the orifice of the renal vein-this is more common from a jugular approach because the veins are directed in a caudalto-cephalad orientation, thus simply advancing the sheath caudally may result in catheterizing one of the veins.

The following are my thoughts on several topics related to filter insertion.

\section{Urgent Filtration}

In my hospital, we consider filter insertion an urgent procedure and will perform it day or night if requested. That being stated, it is important to realize that urgent filtration is controversial and many of my clinical colleagues have variable opinions on this practice. We performed a study to evaluate referral patterns in our institution and found that in 101 consecutive filter insertions, the average elapsed time between the clinical indication for cava filtration and filter placement in all patients was 1.64 days (range, 0 to 17 days). Two patients $(2 \%)$ sustained pulmonary emboli after being diagnosed with deep venous thrombosis before receiving a filter. The lengths of time between filter indication and insertion for these patients were 2 and 8 days.

\section{Choice of Access Site}

I generally prefer the femoral veins for access but there are several advantages of a jugular access. First, the jugular veins are easily accessible using sonographic guidance. Second, patients do not need to remain immobilized after the procedure if a jugular access site is used. Third, when using the Tulip retrievable filter, it can be repositioned several times if it is slightly tilted or not perfectly positioned (this is particularly helpful in a training institution). 


\section{Retrievable versus Permanent Filters}

My threshold for placing a retrievable filter is very low. I place retrievable filters in nearly all younger patients $(<50$ years $)$ and in anyone with any possibility of needing to have it removed, even if I don't plan to remove it over a short time period. This practice is debatable, with many authorities recommending retrievable filter placement only in patients in whom retrieval is planned.

\section{Filters during Venous Thrombolysis}

I do not routinely use filters during deep venous thrombosis thrombolysis. I am not aware of any literature supporting this practice and have not had any patients who have undergone deep venous thrombolysis and developed symptomatic pulmonary emboli in the absence of a prophylactic filter. On the other hand, given the availability of removable filters, I have a low threshold to place one of these devices in the proper clinical context.

\section{Suprarenal Filter Placement}

In both my anecdotal experience and in the limited reported experience, suprarenal filter placement is well tolerated by most patients and should be performed in patients requiring cava filtration who do not have a suitable region below the renal veins for filter placement (e.g., clot in infrarenal inferior vena cava).

\section{Superior Vena Cava Filters}

I have on very rare occasions placed filters in the superior vena cava but we don't offer this routinely in my hospital. In my experience, significant pulmonary embolism from upper extremity deep venous thrombosis is rare.

\section{Symptomatic Filter Occlusion}

In some patients, thrombosis of the inferior vena cava below an indwelling filter results in significant morbidity. We have performed mechanical and pharmacological thrombolysis in a small number of patients with mixed success. Often, organized thrombus is relatively resistant to thrombolysis, requiring protracted treatment. If the cava can be recanalized, symptomatic relief is typical.

\section{SUGGESTED READINGS}

Henkle G, Kunz P, Funaki B. Patterns of referral for inferior vena caval filtration: delays and their impact. AJR Am J Roentgenol 2004;183:1021-1024

Kinney TB. Inferior vena cava filters. Semin Intervent Radiol 2006;23:230-239 